\title{
Efectos del Framing y representaciones sociales de epidemias sanitarias: El Caso de la Gripe A
}

\section{Framing effects and social representations of health epidemics: The case of influenza A}

\author{
Nahia Idoyaga, José Francisco Valencia, Lorena Gil de Montes y Garbiñe Ortiz \\ Universidad del País Vasco / Euskal Herriko Unibertsitatea, España
}

Disponible online 31 de diciembre de 2012

\begin{abstract}
Este artículo recoge una investigación sobre la cobertura y el tratamiento dado por los medios de comunicación a la pandemia de gripe A (H1N1), y la repercusión que ello tuvo en las representaciones sociales sobre la misma. La investigación se basó en los periódicos con mayor tirada de México (país donde se inició) y España durante los meses que duró la crisis en los años 2009-2010. Para ello utilizamos dos marcos teóricos entrelazados: la teoría del Framing o enmarcamiento mediático y la del Colective Symbolic Coping. Los resultados revelaron que el desarrollo de la crisis influyó en el uso de los principales frames o marcos de los medios de comunicación. Además, esos marcos principales pusieron en marcha el proceso de CSC moldeando las representaciones sociales sobre las epidemias sanitarias. De hecho, los cinco clusters principales utilizados para el análisis del CSC (generados por el programa Alceste) se relacionaban con los dos frames más usados: interés humano y atribución de responsabilidad. Finalmente se discuten las implicaciones teóricas y aplicadas derivadas para la investigación sobre la interrelación entre framing, representaciones sociales y rol de los medios de comunicación, así como sobre las estrategias resultantes del discurso de los medios para hacer frente a crisis de salud.
\end{abstract}

Palabras clave: Medios de Comunicación; Gripe A (H1N1); Framing; Collective Symbolic Coping; Representaciones Sociales.

This study analyzed how the mass media covered the influenza A (H1N1) pandemic and its influence on the social representation of the disease. Framing theory and a model of collective symbolic coping were both used to explain the influence of the mass media on social representation. The study was based on analyzing information on the influenza A pandemic provided by national newspapers in Mexico and Spain between 2009 and 2010. The results show that that the development of the crisis affected the use of different kinds of frames in the media. The use of different types of frames led to processes of collective symbolic coping, which are likely to alter the social representation of the epidemic. Data analysis using the Alceste program showed that the most prevalent frames used in the media were human interest and attributing responsibility to institutions. The theoretical implications of this study are discussed in terms of the relationship between framing, social representations and the role of the mass media. Applied implications concern the strategies identified in the media to deal with health crisis.

Key words: Media; Influenza A (H1N1); Framing; Collective Symbolic Coping; Social Representations.

Correspondencia: Nahia Idoyaga. Dpto. de psicología social y metodología de las ciencias del comportamiento. Avda. de Tolosa, 70, 20080 - San Sebastián, Gipuzkoa. Tlf.: 943015680, Fax: 943015670. E-mail: nidoyaga001@ikasle.ehu.es. E-mail de los otros autores: José Francisco Valencia: josefrancisco.valencia@ehu.es; Lorena Gil de Montes: lorena.gildemontes@ehu.es; Garbiñe Ortiz: garbine.ortiz@ehu.es

Los autores desean agradecer la financiación de este trabajo a la ayuda para la formación de investigadores, modalidad predoctoral, concedida por el Gobierno Vasco, así como a la UFI 11/04 de la UPV/EHU 
Entre los acontecimientos que nos rodean hoy en día, las situaciones de riesgo ocupan un lugar preeminente en la simbología social colectiva. Así, al mundo globalizado actual se ha denominado "sociedad del riesgo" (Beck, 1986, 2002, 2006). En este sentido, el relato de los medios de comunicación sobre tales situaciones interviene activamente en dichas constituciones simbólicas.

La presente investigación aborda una de las situaciones de riesgo de mayor impacto mediático y social en los últimos años -el de la Gripe A (H1N1)- analizando el papel de los medios en la construcción simbólica de la misma. Realizaremos dicho análisis entrelazando dos perspectivas teóricas importantes: el Framing y el Colective Symbolic Coping (CSC) y tomaremos como referencias geo-sociales México (país en el que se inició) y España. La Gripe A (H1N1), también conocida como gripe porcina, se detectó por primera vez en marzo del 2009 en México, extendiéndose posteriormente por todo el mundo. Desde ese momento, los medios de comunicación internacionales comenzaron a informar sobre la situación de la gripe y la Organización Mundial de la Salud (OMS) planteó que, por primera vez en la historia, se podría seguir en tiempo real la evolución de una pandemia (OMS, 2009). Países de todo el mundo tomaron medidas extraordinarias para reducir o evitar el riesgo de verse afectados por la gripe, tales como la declaración de alertas sanitarias o el cierre de lugares públicos y se apresuraron a comprar antivirales y vacunas. Sin embargo, desde el verano del 2009 la OMS comenzó a recibir críticas acusándola de crear una alarma excesiva, falta de transparencia, cambiar la definición de pandemia e, incluso, connivencia de intereses con la industria farmacéutica. Finalmente, el 10 de agosto del 2010, la OMS declaró el fin de la pandemia de la Gripe A que, en contraste con su amplia distribución, tuvo una mortalidad baja: 18.449 personas en 214 países (OMS, 2010).

\section{Framing y medios de comunicación}

El modo concreto en que los medios de comunicación encuadran o enmarcan (framing) los acontecimientos y problemas sociales influye en la interpretación y en las actitudes de las personas hacia esos eventos. Sin embargo, más allá del nivel individual, las consecuencias del framing también pueden observarse a nivel social, por su influencia en la opinión pública, la socialización política, y la toma de decisiones colectivas (De Vreese, 2005; Reese, Gandy y Grant, 2001). No obstante, en los procesos de comunicación social los frames no sólo están en los mensajes mediáticos, sino que se encuentran también en el emisor, en el receptor y en la cultura donde aparece el mensaje (Entman, 1993). Es decir, los frames residen tanto en el discurso mediático como en las cogniciones de los individuos, refiriéndose el framing a un proceso de influencia social que conecta los dos (Pan y Kosicki, 2005). En algunos artículos científicos de psicología social suelen utilizarse preferentemente los términos "enmarcamiento" y "marco", mientras que en otros, en la mayoría de hecho, se prefiere utilizar los anglicismos "framing" y "frame". En este artículo se utilizan preferentemente los términos en inglés, aunque a veces también se usan las expresiones en castellano.

Aunque no lo analizó expresamente en relación a la influencia de los medios de comunicación, la definición de frame establecida por Goffman (1974) ha sido comúnmente aceptada. Según este autor, los frames son esquemas de interpretación que permiten a la gente organizar los eventos de la vida en algo que tiene significado. Los frames son, así, principios organizadores, socialmente compartidos y persistentes en el tiempo, que actúan simbólicamente para estructurar u organizar el mundo social (Reese, Gandy y Grant, 2001). Desde esa perspectiva, Entman (1993) estableció las siguientes características del framing mediático: Seleccionar algunos aspectos de una realidad percibida y darles más relevancia en un texto comunicativo, promoviendo así: a) una definición particular del problema, b) una interpretación causal del mismo, c) su evaluación moral y/o d) una recomendación de cómo debe ser tratado.

El campo del framing se ha caracterizado por una cierta confusión conceptual y terminológica. Así, respecto a la relación de la teoría del framing con la agenda-setting y el priming, cabe señalar que si bien algunos autores consideran el framing como un segundo nivel de agenda-setting (McCombs, 2006), existe un creciente consenso en la comunidad científica sobre su autonomía aun considerando su carácter complementario e incluso la utilidad de aplicarlas de forma combinada en los análisis (Kosicki, 2001; Scheufele, 2000; Scheufele y Tewksbury, 2007). La teoría de la agenda-setting comenzó con la idea de considerar que los media no imponen a la opinión pública qué pensar pero sí sobre qué asuntos públicos pensar; cuando las noticias de los media cubren un tema, esto crea en la opinión pública la idea de que el tema es importante. La hipótesis del priming, por su parte, sugiere que la selección de qué noticias aparecen y cuáles se ignoran puede afectar a los juicios que realiza la gente (Iyengar y Kinder 1997); el priming ocurre cuando la gente a la hora de realizar sus evaluaciones, toma en consideración los temas cubiertos recientemente por los media. Así, mientras agenda-setting y priming se relacionan con el "qué" es cubierto por los media, framing se relaciona con el "cómo" lo presentan (Pan y Kosicki, 2005); mientras agenda-setting y priming se basan en la relevancia o prominencia que los medios dan a los acontecimientos, framing se basa en la atribución de las causas (Scheufele, 2000). El prestigioso Journal of Communication dedicó al completo su número 57 (2007) a las cuestiones de Agenda, Framing y Priming, y algunos de los investigadores de mayor prestigio en la materia (Robert Entman, David H. Weaver, David Tewksbury y Dietram A. Scheufele) subrayaban esa doble perspectiva de autonomía y complementariedad.

A pesar de esa diversidad de puntos de vista -o gracias a ella-, la noción de framing ha cobrado en los últimos años un gran impulso en las disciplinas que abordan la comunicación (Vicente y López, 2009), y se presenta como marco teórico tanto para las investigaciones sobre análisis de contenido 
(frame-building) como para las investigaciones sobre la relación entre los medios y la opinión pública (frame-setting) (De Vreese, 2005).

En la investigación sobre framing, se diferencian dos corrientes en relación a los frames mediáticos (De Vreese, 2005; De Vreese, Peter y Semetko, 2001): un enfoque genérico de los frames (generic approach) y un enfoque temático concreto (issue-specific approach). Los frames genéricos son aquellos que transcienden la limitación temática y se pueden utilizar para analizar diferentes hechos, a lo largo del tiempo y en distintos contextos culturales. En cambio, los frames temáticos hacen referencia a hechos concretos con categorías elaboradas de forma específica para ese tema. La línea de trabajo sobre los frames genéricos, revisada y desarrollada por Semetko y colaboradores (Semetko y Valkenburg, 2000; Valkenburg, Semetko y De Vreese, 1999), identifica cinco frames principales que serán los utilizados en este trabajo: conflicto, interés humano, atribución de responsabilidad, moralidad y consecuencias económicas. Diferentes investigaciones han analizado y utilizado esos frames propuestos por Semetko y Valkenburg (2000). De hecho, se ha confirmado que son validos para analizar diferentes temas políticos y sociales, como la inmigración, las crisis, igualdad de género etc. (Ann y Gower, 2009; Igartua et al., 2004; Kenix, 2008; Valencia et al., 2010).

El frame de conflicto refleja las luchas que se puedan dar entre individuos, grupos o instituciones, con objeto de captar el mayor número de audiencia posible. Por ejemplo en el diario El País: China prefiere errar por exceso de precaución y México la acusa de confinar a sus conciudadanos sin fundamento (Reinoso, 2009). Como exponen Semetko y Valkenburg (2000) estudios anteriores habían encontrado que el marco de conflicto era uno de los más utilizados en EEUU, sobre todo en el área de la política, donde por ejemplo, los medios llegaron a ser criticados por incentivar la desconfianza y el cinismo hacia la clase política. En el estudio de estos autores fue el segundo frame más utilizado y en los media más serios aparecía en mayor medida.

El frame de interés humano provee de una cara personal o aspecto emocional a la presentación de un suceso, hecho, o problema. Por ejemplo en el diario El País: Muere la primera persona de H1N1 en España. Dalila Mimouni, marroquí, de 20 años. Estaba embarazada. Un día antes dio a luz un niño. El viudo denuncia mala atención en sus tres visitas a urgencias (Naranjo y Sahuquillo, 2009). A mayor competitividad entre los media, estos tratan de crear un producto que pueda captar y retener el interés público. Ese sería el objetivo de este marco, personalizando las noticias, dramatizándolas o incluyendo emociones. Así, se ha encontrado que este frame influye en las respuestas emocionales de los sujetos, siendo un predictor significativo de la responsabilidad y la culpa atribuidas a las causas del tema o problemática (Cho y Gower, 2006).

El frame de moralidad pone el problema o asunto en el contexto moral o de prescripciones sociales o religiosas. Por ejem- plo, en el diario El País: El que sepa o el que quiera también puede rezar (Ordaz, 2009b). Debido a la norma de objetividad en el periodismo, en general este frame aparece de un modo indirecto, a través de citaciones e inferencias, más que directamente.

El frame de las consecuencias económicas expresa las consecuencias financieras que un problema o asunto puede generar sobre un individuo, grupo, institución, región o país. Por ejemplo, en el diario El Universal: El costo por enfrentar la epidemia del virus de la influenza A H1N1 en México es de 4 mil 306 millones de pesos (Rodriguez, 2009). También este ha sido identificado como un frame común en los media.

Y finalmente, el frame de atribución de responsabilidad, propuesto inicialmente por Iyengar (1990), es definido como un modo de atribuir la responsabilidad de una causa o solución bien al gobierno o a un individuo o grupo. Por ejemplo en el diario El Universal: La Secretaría de Educación Pública (SEP) determinó recorrer el fin del ciclo escolar del 3 al 14 de julio, luego de la suspensión de 9 días de clase ante la contingencia epidemiológica por el virus de influenza A H1N1 (Martinez, 2009). Semetko y Valkenburg (2000) encontraron que la atribución de responsabilidad era el más utilizado en los medios de prensa más serios, mientras los medios más sensacionalistas y la televisión lo utilizaban en menor medida.

Ghang, Faridah y Normah (2010) investigaron el framing que los periódicos malasios hicieron sobre la Gripe A basándose en la escala de Semetko y Valkenburg (2000). Ellos afirmaban que los periódicos son fuente de referencia durante situaciones de crisis por su precio y accesibilidad. Su investigación realzó que el frame significativamente más utilizado fue el de responsabilidad y el menos utilizado el económico, entre ellos quedaron el de moralidad, interés humano y conflicto respectivamente.

\section{Collective Symbolic Coping}

El modelo del CSC explica cómo el grupo le da sentido a situaciones novedosas que amenazan el orden social establecido. Es decir, es el ejercicio que el grupo hace para mantener el mundo tal y como lo conoce, ante una situación nueva o amenazante (Wagner, Kronberger y Seifert, 2002). Inicialmente el coping se refería a la conducta del individuo y no a acontecimientos de nivel colectivo, aunque se reconocía que el esfuerzo individual del coping tiene componentes que son fuertemente sociales y colectivos (Davison y Pennebaker, 1996).

El CSC surge de la interfaz entre la teoría de las Representaciones Sociales (Moscovici, 1988; Wagner y Hayes, 2005) y la Teoría de Agenda-Setting (MacCombs, 2006) subrayando el nivel colectivo del término coping. De la misma manera que en los estudios de clínica, la discusión sobre los problemas tiene efectos sobre el significado que se le da a los traumas (Pennebaker y Harber, 1993). En su intento de proceso para explicar los cambios de los temas de la "esfera pública", el CSC plantea 
que el coping colectivo es creado por los medios de comunicación, quienes construyen y comunican "alguna cosa" como si fuera nueva o como si fuese a cambiar el modo de vida establecido hasta el momento. Siendo parte de una red de comunicación, tanto como individuos como miembros de grupos, las personas valoran el reto que les supone esa nueva cosa (Orr, Sagi y Bar-On, 2000; Wagner, 1998). En consecuencia, las representaciones creadas a través del CSC proveen a la sociedad de herramientas para interpretar nuevos acontecimientos (Gilles et al., 2011). Este proceso, sin embargo, debe cumplir ciertas fases (Wagner et al., 2002):

1) Toma de conciencia (Awareness). Para que haya una toma de conciencia pública de un acontecimiento es necesario que se considere importante para la sociedad (Wagner et al., 2002). Es decir, los medios de comunicación han de darle relevancia a los acontecimientos, encarrilando la toma de conciencia de las personas, es decir, mediante el proceso de agenda-setting al que antes nos hemos referido.

2) Divergencia (Divergence). En esta fase nacen diferentes interpretaciones del acontecimiento creando ambigüedad y confusión. La representación de un nuevo acontecimiento -su modo de conocimiento- puede cambiar el conocimiento previo. Esa novedad de representación y conocimiento puede hacer que fallen los esquemas interpretativos sostenidos hasta el momento y que haga falta uno nuevo.

3) Convergencia (Convergence). Tanto en los medios de comunicación como a nivel individual, las comunicaciones relacionadas al nuevo acontecimiento conllevan que haya una lucha de posibles interpretaciones. Algunas de esas interpretaciones son rechazadas y otras aceptadas (Sperber, 1986), pero al final el grupo llega a un pacto para construir una nueva interpretación aceptada por todos (Bartlett, 1932).

4) Normalización (Normalization). En esta cuarta fase la interpretación o explicación del nuevo acontecimiento se integra en el conocimiento común convirtiéndose en familiar. Sin embargo, la normalización no es irreversible, ya que la discusión política puede reavivar el proceso (Wagner et al., 2002). Además, debemos tener presente que este es un proceso complejo que puede durar décadas y que en ocasiones es difícil de analizar (Bauer, 2000).

En este estudio nos centramos en el uso de las representaciones sociales de la gripe porcina en el enmarcamiento de un tema de riesgo para la salud. ¿Cuál podría ser la relación entre representaciones sociales y enmarcamiento? Los frames y representaciones sociales en primer lugar difieren en el fenómeno al cual se refieren. Las representaciones sociales son interpretaciones socialmente elaboradas sobre un objeto específico, en nuestro caso la gripe porcina. Es a través de las representaciones sociales de la gripe que nosotros comprendemos, damos significado a la "gripe porcina." Frames, por su parte, se relacionan con un tema socio-político específico, en nuestro caso la crisis derivada del manejo (por parte de los estados y las instituciones) del riesgo para la salud. A través del enmarca- miento de dicho tema, los actores tomarán posición al respecto, y definirán qué está en juego y cuáles son los elementos a tomar en cuenta. En consecuencia, un frame tiende a ser más contextualizado y dinámico, relacionándolo con la aparición y resolución (o no) del conflicto (Kaufman y Smith, 1999). A diferencia del enmarcamiento, la representación social de la gripe porcina transciende las cuestiones específicas (salud, economía, etc.).

Por consiguiente, la teoría del CSC es útil para describir los sucesos que amenazan al orden social establecido. Aunque en un principio el modelo del CSC se usó para investigar sucesos relacionados con la biotecnología, hay investigaciones que lo han aplicado en las crisis sanitarias. Así, por ejemplo, en una investigación llevada a cabo sobre la gripe aviar (Gilles et al., 2011) se observó que este modelo era útil para analizar crisis sanitarias, ya que estas, además de suponer una amenaza física, también representaban una amenaza simbólica para la sociedad (Joffe, 1999).

El objetivo principal de este trabajo, en suma, es investigar la cobertura y el tratamiento que los medios de comunicación de México y España dieron a la Gripe A y la repercusión que ese tratamiento tuvo en la sociedad. Se plantean las siguientes hipótesis:

H1) Si bien la visibilidad mediática de este tema disminuiría con el paso del tiempo, se encontrará una correlación positiva entre dicha visibilidad y las amenazas objetivas de la gripe (número de casos, muertos etc.).

H2) Los frames de Responsabilidad, Interés Humano y Conflicto serán los más importantes en los medios de comunicación aunque su presencia irá cambiando durante el tiempo de distinta manera en España y México.

H3) La cobertura de la Gripe A mostrará el desarrollo de las fases del CSC que se verán reflejadas durante esta crisis, aunque es probable que la fase de normalización tenga dificultades de estabilización en la sociedad por el contraste entre percepción y realidad epidemiológica de la pandemia.

\section{Método \\ Muestra y unidad de análisis}

La muestra se extrajo de los diarios de información general de mayor tirada y referencia de México y España: El Universal y El País. El periodo temporal analizado se extendió desde el 25/04/2009 - cuando la Directora General de la OMS, Margaret Chan, en el año 2009, reunió al Comité de Emergencia y éste recomendó que se declarara una "Emergencia de Salud Pública de Interés Internacional"-, hasta el 10/08/2010 cuando declaró el final de la pandemia.

Se establecieron tres unidades de análisis: primero, todas las portadas que hacen referencia a la Gripe A; segundo, los artículos de cualquier sección del periódico a los que se le haga referencia en la portada; finalmente, los editoriales sobre la Gripe A. En total 142 portadas, 142 artículos y 25 editoriales. 


\section{Diseño y codificación}

Se llevaron a cabo los siguientes tres trabajos por separado: A) Análisis de las portadas. Se analizó la cobertura que El País y El Universal hicieron de la Gripe A, situándolo en su espacio temporal y en el contexto epidemiológico.

B) Análisis del framing de los artículos y editoriales. En este apartado se usaron como variables independientes el trimestre en el que se publicó la noticia y el periódico para medir el uso de los cinco frames propuestos por Semetko y Valkenburg (2000) en los artículos y editoriales (en total 167 artículos y editoriales) replicando la escala que establecen los autores para hacerlo en el mismo artículo. La codificación fue llevada a cabo por dos jueces ciegos obteniendo un alto índice de acuerdo (Kappa de Cohen $>$.70).

C) Análisis de contenido de los artículos y editoriales. Se analizaron los artículos y editoriales para determinar los principales temas tratados. Para evitar los problemas de fiabilidad y validez comunes en los análisis de texto se optó por usar como instrumento el programa Alceste que realiza clasificación textual automática (Bauer, 2000; Klein y Likata, 2003; Reinert, 1996). El objetivo de este software es cuantificar un texto para extraer sus estructuras más importantes con el fin de aprovechar la información esencial contenida en los datos textuales. Se ha demostrado (Reinert, 1996) que estas estructuras están estrechamente vinculados a la distribución de las palabras en un texto y que esta distribución rara vez se hace al azar. Describir, clasificar, asimilar, sintetizar automáticamente un texto son los intereses de Alceste.

\section{Resultados \\ Situación en tiempo y contexto}

Para comenzar se analizaron mes a mes las portadas y los artículos publicados en el diario español El País sobre la Gripe A y el número de casos y fallecimientos a causa de la misma en España. El análisis de correlación de Spearman encontró una correlación significativa entre los artículos publicados en El País y los casos de Gripe A en España $r_{(17)}=.567$ $p<.05$, aunque cabe destacar que el $40 \%$ de los artículos se publicaron antes de que la Gripe A llegara a España. A partir de las encuestas realizadas durante esos meses por el Centro de Investigaciones Sociológicas (CIS) se analizó también en qué medida percibieron los españoles la salud como un problema. Por una parte, se encontró una correlación positiva entre el número de artículos publicados y el grado en que los españoles percibieron la salud como un problema $r_{(17)}=.58, p<.05$, hecho que confirma la relación entre el efecto de la agenda de los medios y la agenda pública. Igualmente, se encontró una correlación positiva entre esos resultados del CIS y el número de casos $r_{(17)}=.61, p<.05 \mathrm{y}$ defunciones $r_{(17)}=.52, p<.05$ por Gripe A. También es de destacar, que los meses donde hubo más presencia de la Gripe A en la prensa (de abril del 2009 a diciembre del 2009) la percepción de la sanidad como un problema fue media de 4.6 puntos, cuando en el periodo anterior comprendido de de mayo del 2009 a marzo del 2009 había sido de 4 puntos y el periodo posterior comprendido de enero del 2010 a diciembre del 2010 de 3.5 puntos. Luego, los españoles consideraron que la salud representaba un problema en mayor medida cuando el tema de la Gripe A tenía más presencia en los medios.

En relación al diario El Universal, y analizando nuevamente mes a mea las portadas y los artículos publicados en el mismo sobre la Gripe A y el número de casos y fallecimientos producidos por ella en México, los análisis de correlación de Spearman encontraron correlaciones significativas entre los artículos y los casos de Gripe $\mathrm{A} r_{(17)}=.85, p<.01$; y los artículos $\mathrm{y}$ defunciones a causa de la Gripe A $r_{(17)}=.90, p<.01$. También se encontraron correlaciones significativas entre las portadas y el número de casos de la Gripe A $r_{(17)}=.80, p<.01$ y entre las portadas y el número de defunciones $r_{(17)}=.75, p<.01$. Así, en México la publicación de portadas o titulares y artículos sobre la Gripe A estuvo directamente relacionado al número de afectados por la misma.

\section{Análisis del framing de los artículos y editoriales}

En total se analizaron 142 artículos (a los que se les había hecho referencia en las portadas) y 25 editoriales, perteneciendo el $39.5 \%$ a El País y el $60.5 \%$ a El Universal. La Tabla 1 muestra que el frame más utilizado fue el de interés humano, seguido por el de responsabilidad, conflicto, moralidad y por último por el económico.

Tabla 1

Media y desviación típica de los frames utilizados

\begin{tabular}{lcc}
\hline \multicolumn{1}{c}{ Frame } & $M$ & $D T$ \\
\hline Interés humano & 1.75 & 0.66 \\
Responsabilidad & 1.64 & 0.50 \\
Conflicto & 1.59 & 0.63 \\
Moralidad & 1.54 & 0.51 \\
Económico & 1.46 & 0.75 \\
\hline
\end{tabular}

Nota: $M=$ Media; $D T=$ Desviación típica.

\section{Frame, Tiempo y Periódicos}

En relación a El País, se realizaron ANOVAs correspondientes a cada tipo de frame (ver Gráfico 1) y se encontraron diferencias significativas por trimestre en los frames de Interés Humano $F_{(4,61)}=5.10, p<.001\left(h_{p}^{2}=.25\right)$, Conflicto $F_{(4,61)}=2.40$, $p<.05\left(h_{p}{ }^{2}=.136\right)$ y Económico $F_{(4,61)}=4.13, p<.001\left(h_{p}{ }^{2}=.213\right)$, no, sin embargo, en los frames de Responsabilidad $F_{(4,61)}=1.30$, $p>0.5$ ni Moralidad $F_{(4,61)}=0.89, \mathrm{p}>0.5$.

Las pruebas DSM sobre el frame de interés humano revelaron que el primer trimestre era significativamente diferente del segundo $(p \leq .001)$, tercero $(p \leq .01)$, cuarto $(p \leq .01)$ y sexto $(p \leq .01)$. Es decir, al principio se usó mucho el frame de interés humano ya que se esperaba que la de la Gripe A fuera una epidemia muy virulenta. Pero desde el segundo trimestre en adelante, debido a que la repercusión no era tan grave, el uso de este frame disminuyó. 
Figura 1

Uso de los principales frames por trimestre en El País y El Universal.
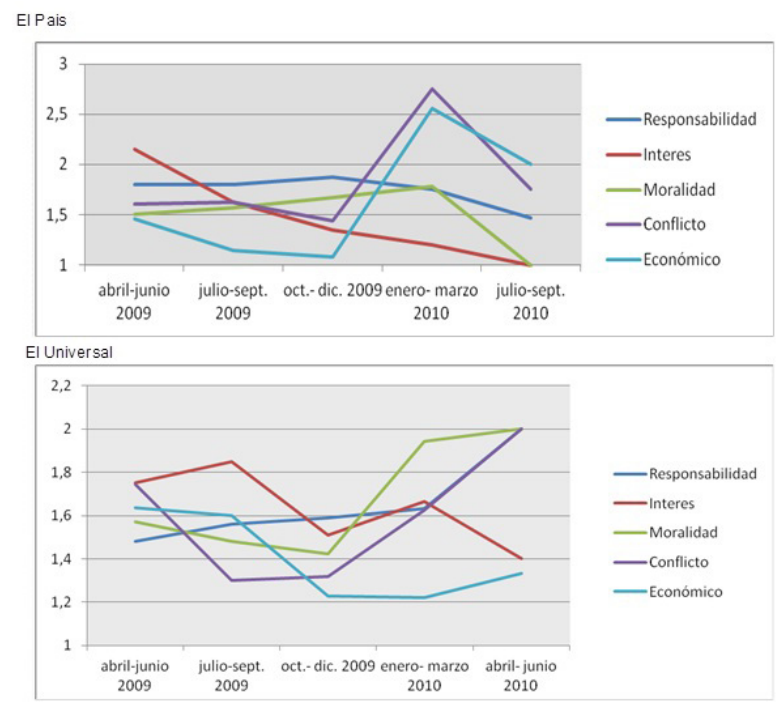

En el frame de conflicto, las pruebas DSM mostraron que el cuarto cuatrimestre es significativamente diferente al primero ( $p \leq .001)$, segundo $(p \leq .001)$ y tercero $(p \leq .01)$. La razón de ello es que en España la principal "discusión" sobre la Gripe A comenzó a finales de la crisis (desde el cuarto trimestre en adelante). En ese conflicto, se discutieron la idoneidad de la respuesta dada por las instituciones ante la crisis de la Gripe A y el gasto económico que supuso. Finalmente, las pruebas DSM del frame económico encontraron que el cuarto trimestre era, también, significativamente diferente del primero $(p \leq .001)$, segundo $(p \leq .001)$ y tercero $(p \leq .001)$. De hecho, este frame está especialmente ligado al frame de conflicto ya que según varios sectores de la sociedad en la crisis de la Gripe A se gastó demasiado dinero institucional a favor de intereses empresariales particulares. Aun así al interpretar tanto estos últimos datos como los del frame de conflicto deberá tenerse en cuenta que durante este cuarto trimestre la presencia de artículos sobre la Gripe A es bastante menor que en los tres trimestres anteriores por lo que los resultados podrían estar condicionados.

En relación a El Universal los análisis ANOVA correspondientes a cada tipo de frame (ver Figura 1) solamente encontraron diferencias significativas por trimestre en el frame de conflicto $F_{(4,96)}=3.74, p<.001\left(h_{p}^{2}=.135\right)$. En este caso, las pruebas DSM mostraron diferencias significativas entre el primer trimestre y el segundo ( $p \leq .00)$ y el tercero $(p \leq .00)$. Básicamente, en México hubo dos momentos de conflicto: el primero en los primeros meses de la crisis debido al rechazo sufrido por parte de ciertos países (EEUU y países centroamericanos plantearon el cierre de fronteras, en Francia y Singapur los aviones provenientes de México tuvieron problemas para desembarcar etc.) y el segundo a partir del cuarto trimestre en adelante, tal y como pasó tanto en España como a nivel global, debido a que es cuando comenzó el debate sobre la respuesta dada a la crisis y el gasto económico que conllevó.

\section{Análisis de contenido de los artículos y editoriales}

El análisis de contenido de los artículos y de los editoriales se realizó mediante el programa Alceste. El corpus (129.462 palabras, de las cuales 11.561 eran palabras distintas) incluyó aquellos artículos que hacen referencia a la gripe A y son mencionados en las portadas de los periódicos y todos los editoriales. De acuerdo con el proceso del Alceste (Boudes y Cellier, 1998), el vocabulario caracterizador de las clases se dividió en subclases, para ello solamente se analizan las 'palabras completas' (nombres, verbos, adjetivos, adverbios); 'las palabras de herramienta' (artículos, pronombres, conjunciones) se excluyen. Esas 'palabras completas' se reducen a su raíz (ej. 'mex' incluye mexicano, México, etc.). En concreto, el análisis jerárquico descendente dividió el corpus en 3.142 Unidades Contextuales Elementales (ECUs) de las que se trabajaron 1.933 (62\%) y extrajo 5 clases de ECUs (ver Figura 2). La primera clase obtuvo 364 ECUs (19\%), la segunda 505 ECUs (26\%), la tercera 484 ECUs (25\%), la cuarta $201(10 \%)$ y la quinta 379 ECU $(20 \%)$.

\section{Figura 2}

Dendograma de las clases generadas por la Clasificación Jerárquica descendente

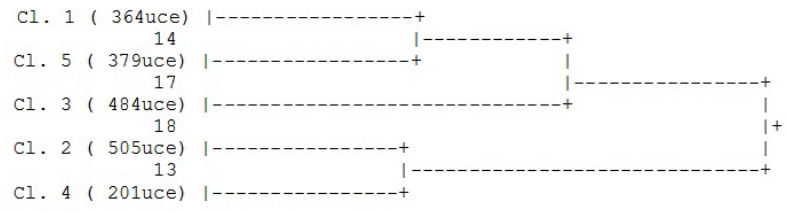

\section{Contenido de las clases}

Para extraer las clases principales del Alceste el vocabulario ligado a cada uno de ellos se dividió en subclases (la frecuencia de cada palabra o raíz aparece entre paréntesis):

Clase 1: Prevención para protegerse de la gripe.

1) Partes del cuerpo: mano (144), sexual (52), boca (48), sus (45), beso (39), estornud (38)...

2) Herramientas de prevención: mascarilla (80), lavarse (48), enfermer (45), cubreboca (42), guantes (41), pañuelo (39)...

3) Empresas: empresa (85), emple (84), trabaj (76), trabajador (63), cliente (58)

4) Otros: plan (59), contingencia (50), frecuencia (38), evitar (37)...

Clase 2: La pandemia global y la respuesta de la OMS

5) Gripe A: virus (207), pandemia (138), mutación (107), aviar (87), infect (69) h5n1 (66), nueva (58), gripe (49)... 6) OMS: OMS (215), chan (86), fukuda (64), declar (46)...

7) Globalidad: expansión (66), fase (51), alerta (46), mundo (44)...

8) Otros: caus (56), leve (56), parec (48)... 
Clase 3: Creación y aplicación de vacunas.

9) Vacunas creadas: vacun (703), dosis (246), millón (163), vacunación (99), aplic (86), produc (68), lote (57), estacional (53), compr (56), medicament (50), fármaco (44)...

10) Farmacéuticas: laborator (206), grupo (83), farmaceut (56), sanofi (54), ensayo (51), birmex (47), investigad (44)...

11) Otros: diciembre (46), riesgo (43), octubre (41)...

Clase 4: Muertos y víctimas de la Gripe A

12) Afectados mortales: deceso (152), fallecimiento (95), muert (90), defunción (80) fallec (71), muer (78)...

13) Registro de afectados: caso (307), report (253), registr (171), contabiliz (155), confirm (152), numero (126), cifra (115), neumonia (82), ultim (75), total (58), aument (55), contagio (47), pico (46)...

14) Otros: ssa (106), increment (104), agosto (79)...

Clase 5: Gestión gubernamental de la crisis

15) Actividades que se paralizaron: clase (195), escuela (173), educación (140), suspend (133), actividades (119), escolar(101), acción(63), vigilancia (59), medida (55)...

16) Mandatarios: secretar (190), angel (131), cordova (122), jose (99), calderón (99), felipe (79), ebrad (70), ahued (56), villalobos (55)...

17) Otros: plantel (66), epidemiolo (66), regres (55)...

\section{Relaciones con las variables independientes}

Por medio de los test de independencia el Alceste computa la relación entre las clases léxicas y los niveles de una variable independiente. Si se da una mayor proporción significativa de ECUs que pertenecen a una clase a un nivel de la variable independiente más que a otros niveles combinados, la clase se considera asociada a este nivel. El programa ejecuta el mismo análisis para todas las otras clases y niveles de la variable independiente.

En nuestro caso se clasificaron los artículos en función de los periódicos. El País se relacionó con la primera $X_{(1)}^{2}=26.81$, $p<.001$ y la segunda clase $X_{(1)}^{2}=211.70, p<.001$. El Universal, sin embargo, con la tercera $X^{2}{ }_{(1)}=15.22, p<.001$, cuarta $X_{(1)}^{2}=41.11$, $p<.001$ y quinta clase $X_{(1)}^{2}=126.81, p<.001$. En suma, se puede concluir que el discurso de El Universal se centró en la respuesta dada a la Gripe A en México, mientras que el de El País opto por tratar el tema de una manera más global.

Otra variable analizada fue el mes de publicación del artículo o editorial. En la tabla 2 se puede observar cómo desde abril del 2009 a febrero del 2010 hay una gran afluencia de discursos con la presencia de todas las clases, pudiendo entenderse, en términos de CSC, como la fase de toma de conciencia el primer trimestre y la de divergencia el segundo. Sin embargo desde febrero la cantidad de discursos sobre la Gripe A descendió significativamente pudiendo entenderse como la fase de convergencia, y en agosto, cuando se dio por finalizada la pandemia, sólo apareció una de las clases (La pandemia global y la respuesta de la OMS) de las presentadas anteriormente.

\section{Discusión \\ Presentación de la crisis en los periódicos}

La crisis de la Gripe A tuvo una presencia considerablemente alta en la prensa desde abril a octubre del 2009, siendo portada de los periódicos de ambos países uno de cada cuatro días y habiendo de medía dos artículos sobre este tema todos los días en cada periódico. Sin embargo desde ese momento en adelante la discusión pública sobre la Gripe A fue perdiendo presencia según el examen efectuado a las portadas de los periódicos. Basándonos en la teoría de agenda-setting (McCombs y Shaw, 1972) podemos afirmar que durante esos meses la Gripe A tuvo un gran eco en la sociedad y que la visibilidad del problema (Etnman, 1993; McCombs, Lopez y Llamas, 2000), es decir, la discusión sobre la gripe, fue disminuyendo en la esfera pública con el paso del tiempo, confirmando así nuestra primera hipótesis. Además, los análisis de correlación demostraron que el número de artículos publicados tenía relación con la amenaza objetiva. Dicho de otra manera, que se encontró una correlación significativa y positiva entre el número de publicaciones y casos de Gripe A, tanto en El País como en El Universal: cuanto mayor era el número de casos, se publicaban más artículos. Estas correlaciones fueron mayores en El Universal $(r=.85)$ que en El País $(r=.56)$, es decir, en

Tabla2

Frecuencias de las clases por mes

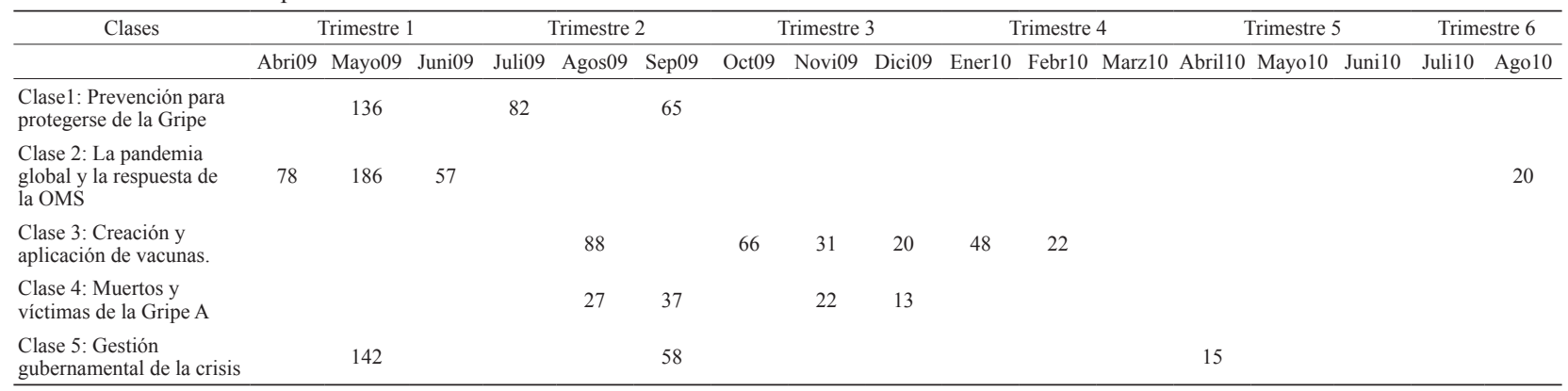

Nota: En todos los casos: $\left.X^{2}(d f=1) \geq 5 ; p=.001\right)$. 
el contexto donde la amenaza objetiva era mayor, en México, el seguimiento por parte de los medios de comunicación fue mayor.

Si se compara la cobertura de este tema en El País (España) y El Universal (México) lo primero que se debe analizar es el contexto temporal de su publicación. La mayoría de artículos de El País se publicaron antes de que la Gripe A llegase a España, es decir, el hecho de que irrumpiera una epidemia en México, aun cuando no había víctimas en España, fue un acontecimiento noticioso relevante para la prensa de referencia española. Por ejemplo en el diario El País: Alarma mundial por el virus: La OMS ve "muy grave" el brote de gripe porcina en México (Ordaz, 2009a). Sin embargo en México hubo una correlación más alta entre los artículos publicados y el número de casos. Se puede decir, por ello que la prensa mexicana realizó una cobertura de la crisis que estaban viviendo en ese momento. Los medios españoles, en cambio, publicaron sobre una crisis hipotética que pudiera que llegara al país.

\section{Framing: El discurso sobre la Gripe A en los periódicos}

La segunda cuestión analizada es el tratamiento de la Gripe A por parte de los periódicos. Primeramente, examinando los frames propuestos por Semetko y Valkenburg (2000) se demuestra que se dio un uso condicionado de los mismos. Así, el frame más utilizado ha sido el de Interés Humano, seguido por el de Atribución de Responsabilidad, Conflicto, Moralidad y por último el Económico. En otras palabras, el uso de estos frames demuestra que la Gripe A se definió como un acontecimiento de consecuencias graves para los ciudadanos, donde los mandatarios deben asumir la responsabilidad para hacer frente al problema, confirmando también nuestra segunda hipótesis.

Debemos resaltar que aunque el orden de los frames más utilizados de nuestra investigación no concuerda con otras investigaciones realizadas en el mismo campo (Chang, 2010) si que se repite una idea principal, el uso relevante del frame de responsabilidad y el poco uso del frame económico. El uso del frame de Interés Humano, especialmente, fue muy elevado al inicio de la crisis. Por ejemplo en el diario El Universal: María llevó a su hija de 5 años, con fiebre y dolores musculares, a dos hospitales, donde le recetaron paracetamol y diagnosticaron fractura de fémur; horas después, la menor murió (Piña, 2009). Aunque con el tiempo disminuyó y fue dejando lugar a otros frames. Investigaciones hechas en este campo muestran que este frame influye en la respuesta emocional de las personas, prediciendo la atribución de responsabilidad o culpabilidad del tema tratado (Cho y Gower, 2006). Esto nos induce a pensar que el segundo frame más utilizado sea el de Atribución de Responsabilidad, es debido al deseo de responder a la situación creada por el interés humano. Además, recordemos que es común que los medios de comunicación se fijen en la Atribución de Responsabilidad cuando los actores principales (gobierno, mandatarios internacionales, OMS, etc.) tienen un grado alto de control (Ann y Gower, 2009).
Frecuentemente el frame de Atribución de Responsabilidad conlleva información de movilización que ayuda a las personas a tomar decisiones de actuación o prevención ante un riesgo (Tanner et al., 2009) como el que pudo ser la Gripe A. Por ejemplo en el diario El Universal: Prevén detener toda la actividad en el DF: Cierran guarderías y tribunales locales (Archundia, 2009). Estos resultados son acordes a los planteados por Faridah, Normah y Chang (2010), quienes afirmaban que en situaciones de crisis o catástrofes, especialmente cuando hace falta una respuesta inmediata (por ejemplo para hacer frente a la expansión de la Gripe A) el uso de este frame puede ser decisivo para hacer llegar a la población la información necesaria. Además, llama la atención que el uso del frame de atribución de responsabilidad no cambia durante el tiempo. Que este frame se mantenga elevado durante toda la crisis, posibilitó que organizaciones como la OMS tuvieran una especial relevancia durante esta pandemia (Rocamora, 2012). Además este frame se distribuyó de forma parecida en los dos países, manteniéndose en nivel alto durante toda la crisis.

Respecto al frame de Conflicto, como ya se ha dicho anteriormente, apareció en dos momentos. Una primera vez en México, debido al rechazo sufrido por parte de otros países. Por ejemplo en el diario El Universal: Crece rechazo en el mundo a mexicanos (Rosas, 2009). Y al final de la crisis a nivel mundial por el debate sobre la gestión de la pandemia hecha por la OMS. Por ejemplo en el diario El País: Fisuras en la causa contra la OMS: El Consejo de Europa, dividido ante la gripe A (De Benito, 2010). Algunos autores (Rocamora, 2012) han definido este segundo momento de conflicto con el comienzo de una comunicación de crisis, donde el discurso de la OMS se basó en la justificación de sus propios actos más allá de la comunicación sobre la Gripe A.

Por último es interesante que los medios de comunicación no dieran tanta importancia a las repercusiones morales y económicas de la gripe. Aunque no dar mucha importancia a las consecuencias morales sea bastante común (Igartua et al., 2004; Semetko y Valkenburg, 2000; Valencia et al., 2010) en el caso de las consecuencias hay diversidad de resultados. La investigación ha encontrado que en temas como el de la inmigración era el frame más usado (Igartua et al., 2004) mientras no es así en otros temas, como el caso de la igualdad de género (Valencia et al., 2010). Por otro lado, profundizando en el significado del framing, además de los resultados de la propuesta de Semetko y Valkenburg (2000), se encontraron resultados similares con el análisis ofrecido por el programa Alceste.

De hecho, usando dicho método se demostró que todas las clases se ligaban al frame de Interés Humano (Muertos y víctimas de la Gripe A, La pandemia global y la respuesta de la OMS) o de Atribución de Responsabilidad (Gestión gubernamental de la crisis, Creación y aplicación de vacunas, Prevención para protegerse de la gripe). Además, como ya se encontró en el análisis del framing, las clases que representaban el Interés Humano se relacionaban con los dos primeros trimestres, dis- 
minuyendo con el tiempo. Cabe destacar también que mediante el programa Alceste se puede observar de una manera más precisa el protagonismo de la OMS en el discurso de los periódicos, confirmando, con ello, que los altos niveles de poder tienen una manifiesta influencia en este frame (Ann y Gower, 2009).

\section{EI CSC en los periódicos}

Tanto en México como en España durante el primer trimestre de la crisis (abril, mayo y junio del 2009) la Gripe A tuvo una presencia muy alta en los periódicos, apareciendo en la portada una de cada dos días. Esto, siguiendo el proceso de agenda-setting, lo convirtió en acontecimiento relevante para la sociedad fomentando la toma de conciencia. De hecho, como confirmaron los índices objetivos publicados por los barómetros mensuales del CIS, hubo una correlación positiva entre la cobertura de los medios de comunicación y la preocupación de la sociedad por la salud. Este sería un claro reflejo del proceso de toma de conciencia. Desde ese momento en adelante, es decir de julio del 2009 a febrero del 2010 podemos observar características de la fase de divergencia, ya que en esa época hubo una gran diversidad de clases. De hecho, aparecen todas las clases encontradas en los artículos: los ligados a la gestión de los gobiernos, a organizaciones internacionales, a vacunas, sobre empresas etc.

Llama la atención, como podemos ver en el análisis de conglomerados de la figura 2, que en esa fase de divergencia la segunda y cuarta clase aparezcan unidas. Es decir, "La pandemia global y la respuesta de la OMS" y "Muertos y víctimas de la Gripe A" aparecen como discursos ligados en la distribución del clúster. Estos discursos hacen que la Gripe A se relacione con situaciones de peligro o miedo mediante el uso de términos como pandemia o muerte. Esto concuerda con investigaciones centradas en la influencia de esa trasmisión de miedo o peligro en las representaciones sociales (Altheide, 2010; Gonzalo y Farré, 2011).

En lo que respecta a la fase de convergencia, y como ocurría en otras investigaciones (Gilles et al., 2011), no se refleja de manera tan clara como las fases anteriores. Aun así, podríamos decir que aparece de marzo del 2010 en adelante, con la bajada de cobertura y la desaparición de marcos inconsistentes. Más claro todavía, en agosto del 2010, en el discurso resultante cuando la OMS declaró la pandemia finalizada, donde se podía atisbar un cierto acuerdo sobre la epidemia y sus efectos. Cabe destacar que el único discurso que duró hasta esa época fue "La pandemia global y la respuesta de la OMS", que como ya se ha mencionado está ligado a la transmisión de miedo o peligro. Luego, creemos que es necesario investigar más profundamente si este tipo de discurso influye en la creación de la representación social de que vivimos en una "sociedad de riesgo" (Beck, 1986, 2002, 2006). Por otra parte, al igual que en otras investigaciones sobre crisis epidemiológicas (Gilles et al., 2011) no se detectó la fase de normalización. En su lugar se observó una fase que podríamos denominar de "situación para- dójicamente normalizada" (Rosenbrock et al., 2000; Setbon, 2000), en la que aparecieron desacuerdos en la sociedad entre la percepción de peligro y la realidad epidemiológica de la crisis. Esto se podría justificarse por los pronósticos hechos antes de la pandemia o por padecer el riesgo de epidemia con una frecuencia relativamente asidua. De hecho, las representaciones creadas de epidemias anteriores pueden tener efecto en la fase de toma de conciencia de los nuevos acontecimientos (Gilles et al., 2011). Todo ello confirma nuestra tercera hipótesis.

Resumiendo lo anterior, podemos afirmar que los discursos creados mediante el framing de los medios de comunicación tienen un efecto directo en las representaciones sociales mediante el proceso del CSC. Además la teoría del CSC complementa a la de agenda-setting, por la influencia que tiene la cantidad de artículos publicados en la opinión pública (medida por la pregunta "el problema de la salud" del barómetro del CIS). En este sentido estos resultados confirman igualmente la solidez de la hipótesis central de la teoría de la agenda-setting según la cual los medios de difusión transfieren al público, la percepción de los problemas sobre los cuáles se ha de fijar la atención, como su propia importancia. Por otra parte, se confirma igualmente la relación entre la amenaza subjetiva (el problema de la salud) y la amenaza objetiva (número de casos, tasa de mortalidad y las correlaciones diferentes de ambos países), así como con las fases de CSC que aparecen a lo largo del periodo de la crisis. En este sentido, y a nivel aplicado, como el uso de los frames influye en la percepción de riesgo y la respuesta a la misma que dan las personas, sería muy importante impulsar una eficaz política comunicativa sanitaria en estos casos. Además, sería muy positivo que esas comunicaciones llegaran a la fase de normalización mencionada en el CSC, convirtiéndose así en fuentes de conocimiento para posibles futuras epidemias sanitarias. Esta estrategia de investigación puede ayudar a superar los problemas de determinismo social y reduccionismo cognitivo planteados para la teoría de las representaciones sociales (Voelklein y Howard, 2005) y los problemas de relación entre la versión deductiva -enfoque genérico- y la inductiva -enfoque temático- de la teoría del framing (De Vreese, 2005). Por una parte, la utilización conjunta de la estrategia de Semetko y Valkenburg y del análisis Alceste puede ayudar a integrar ambas versiones, y por otra, la relación entre el discurso de los media y la percepción del publico pueden ayudar a plantear una visión más dinámica y social para los problemas de la teoría de las representaciones sociales. En este estudio esta segunda estrategia es la más débil. Debido al "experimento natural" del surgimiento y desarrollo del problema de la gripe porcina, solamente se ha tenido acceso a la utilización de los datos de los barómetros y no por ejemplo a la utilización de entrevistas grupales u otro tipo de estrategias de investigación como se ha realizado en otros estudios (ej. Biotecnología Wagner et al., 2002). No es menos cierto, sin embargo, que el objetivo básico de este estudio no pretendía sino un primer acercamiento a la relación entre el framing y 
el CSC. En este sentido, y para finalizar, pensando en investigaciones futuras, sería muy importante reflexionar sobre las consecuencias del framing. Los frames son herramientas que nos ayudan a transmitir, interpretar y evaluar el mundo social (Van Gorp, 2007) y mediante ellos además de dar información sobre "el problema" los medios de comunicación también nos dicen cómo interpretarlo. A la hora de continuar con esta investigación, sería interesante analizar el framing como variable independiente (De Vreese et al., 2011), introduciéndolo en un diseño experimental. Es decir, analizar la influencia de las noticias de la prensa en las opiniones de la gente sobre esta crisis sanitaria (frame-setting) así como la influencia de los diversos tipos de crisis y pandemias (De Vreese et al., 2011). Por otra parte, sería interesante también complementar los framings creados deductivamente por Semetko y colaboradores con los framings creados inductivamente. En concreto, complementar estos frames (generic frames) con los resultados de los frames derivados inductivamente (issue-specific news frames). Esto puede posibilitar profundizar, por una parte, en el significado del framing (analizando las características de los actores y el espacio de esta investigación) y por otra, en la interrelación teórica entre representaciones sociales y framing (por medio del programa Alceste, aquí realizado).

\section{Referencias}

1. Altheide, D. (2010). Risk communication and the discurse of fear. Catalan Journal of Communication \& Cultural Studies, 2, 145-158. http://dx.doi.org/10.1386/cjes.2.2.145 1

2. Archundia, M. (2009, 27 de abril). Prevén detener toda la actividad en el DF. El Universal, pag. 4-14

3. Ann, S.K. y Gower K. (2009). How do the news media frame crises? A content analysis of crisis news coverage. Public Relations Review, 35, 107-112. http://dx.doi. org/10.1016/j.pubrev.2009.01.010

4. Bartlett (1932). Remembering. Cambridge: Cambridge University Press.

5. Bauer, M. W. (2000). Science in the media as cultural indicator: Contextualizing surveys with media analysis. En M. Dierkes y C. Von Grote (Eds.), Between understanding and trust: the public, science and technology (pp. 157-178). Amsterdam: Routledge.

6. Bauer, M., Gaskell, G. y Durant, J. (2001). The years of controversy: Biotechnology 1996-1999. London: Museum of Science and Industry.

7. Beck, U. (1986). Risikogesellschaft-Auf dem Weg in eine andere Moderne. Frankfurt: Suhrkampl.

8. Beck, U. (2002). La sociedad del riesgo global. Madrid: Siglo XXI de España Editores.

9. Beck, U. (2006). La sociedad del riesgo: hacia una nueva modernidad. Barcelona: Paidós Ibérica.

10. Chan, M. (2009). El nivel de alerta de pandemia de gripe se eleva de la fase 5 a la fase 6. Documento consultado el 14/03/12. Disponible: http://www.who.int/mediacentre/ news/statements/2009/h1n1_pandemic_phase6_20090611/ es/index.html.

11. Cho, S. y Gower, K. K. (2006). The Effect of Framing on Public's Perception of Crisis: Human Interest Frame Effect on Attributions to Responsibility and Blame. Trabajo presentado en Annual meeting of the International Communication Association. 19-23 Junio, Dresden.

12. Davison, J. y Pennebaker, J.W. (1996). Social psychosomatics. En: E.T Higgins \& A.W. Kruglanski (Eds.), Social psychology: Handbook of basic principies (pp. 102-132). New York: Guilford Press.

13. De Benito, E. (2010, 23 de Enero). Fisuras en la causa contra la OMS. El País, pag.1

14. De Vreese, C.H. (2005). News framing: Theory and typology. Document Design, 13, 51-62.

15. De Vreese, C. H., Boomgaarden, H. G. y Semetko, H. A. (2011). (In)direct framing effects: The effects of news media framing on public support for Turkish membership in the European union. Communication Research, 38, 179205. http://dx.doi.org/10.1177/0093650210384934

16. De Vreese, C. H., Peter, J. y Semetko, H. A. (2001). Framing politics at the launch of the euro: A crossnational comparative study of frames in the news. Political Communication, 18, 107-122. http://dx.doi. org/10.1080/105846001750322934

17. Entman, R. (1993). Framing: Toward Clarification of a Fractured Paradigm. Journal of Communication, 41, 51-58. http://dx.doi.org/10.1111/j.1460-2466.1993.tb01304.x

18. Faridah I., Normah M. y Chang P. (2010). Framing a pandemic: analysis of malaysian mainstream newspapers in the 1 1n1 coverage. Paper presented at International Communication Association 2010 Preconference - Health Communication Campaigns: Issues and Strategies in Asia, Australia and Southeast Asia. Singapore.

19. Ghang P., Faridah I. y Normah M. (2010). Framing a pandemic: analysis of malaysianmainstream newspapers in the H1NI coverage. Journal of Media and Information Warfare, 3, 105-122

20. Gilles, I., Bangerter, A., Clemence, A., Green, E.G.T., Krings, F., Mouton, A., Rigaud, D., Staerkle, C. y WagnerEgger, P. (2011). Collective symbolic coping with disease threat and othering: A case study of avian influenza. British Journal of Social Psychology. http://dx.doi.org/10.1111/ j.2044-8309.2011.02048.x

21. Goffman, E. (1974). Frame Analysis: An Essay on the Organization of Experience. New York: Harper \& Row.

22. Gonzalo J.L. y Farré, J. (2011). Teoría de la comunicación de riesgo. Barcelona: Editorial UOC.

23. Igartua, J. J., Humanes, M. L., Muñiz, C., Cheng, L. Mellado, C., Medina, E. y Erazo, M. A. (2004). Tratamiento informativo de la inmigración en la prensa española y opinión pública. En A. Verano (Comp.), Setenta años de periodismo y comunicación en América Latina. Buenos 
Aires: Ediciones Universidad de La Plata.

24. Iyengar, S. (1990). Framing responsability for political issues: The case of Poverty. Political Behavior, 12, 19-40. http://dx.doi.org/10.1007/BF00992330

25. Iyengar, S. y Kinder, D. R. (1997). News that matters: Television and American public opinion. Chicago: University of Chicago Press.

26. Joffe, H. (1999). Risk and the "other". Cambridge: Cambridge University Press.

27. Kaufman, S. y Smith, J. (1999). Framing and Reframing in Land Use Change Conflicts. Journal of Architectural and Planning Research, 16, 164-180.

28. Kenix, L.J. (2008). From media frame to social change? A comparative analysis of same-sex rights in the United States and New Zealand press. Australian Journal of Communication, 35, 105-128.

29. Klein, O. y Licata, L. (2003). When group representations serve social change: The speeches of Patrice Lumumba during the Congolese decolonization. British Journal of Social Psychology, 42, 571-593. http://dx.doi. org/10.1348/014466603322595284

30. Kosicki, G. M. (2001). The media priming effect: News media and considerations affecting political judgments. En J. P. Dillard y M. P. Pfau (Eds.), The persuasion handbook: Developments in theory and method (pp. 63-81). Thousand Oaks, CA: Sage Publications.

31. Martínez, N. (2009, 12 de mayo). Entidades que no reiniciaron ayer harán ajuste local, plantea Lujambio. El Universal, pág. 4-14

32. McCombs, M. (2006). Estableciendo la agenda. El impacto de los medios en la opinión pública y en el conocimiento. Barcelona: Paidós Comunicación.

33. McCombs, M., Lopez-Escobar, E. y Llamas, J. P. (2000). Setting the agenda of attributes in the 1996 spanish general election. Journal of Communication, 50, 77-92. http:// dx.doi.org/10.1111/j.1460-2466.2000.tb02842.x

34. McCombs, M.E. y Shaw, D.L. (1972). The Agenda-Setting Function of Mass Media. Public Opinion Quarterly, 36, 176-187. http://dx.doi.org/10.1086/267990

35. Moscovici, S. (1988). Notes towards a description of social representations. European Journal of Social Psychology, 18, 211-250. http://dx.doi.org/10.1002/ejsp.2420180303

36. Naranjo, J. y Sahuquillo, M.R. (2009, 10 de Julio). Diario del H1N1 en España. El País, pag. 31

37. OMS (2009). Recurso a órganos consultivos por la OMS en su respuesta a la gripe pandémica (Nota informativa $\mathrm{N}^{\circ}$ 19). Documento consultado el 14/03/12. Disponible: http://www.who.int/csr/disease/swineflu/notes/briefing 20091203/es/index.html

38. OMS (2010). Respuesta internacional a la pandemia de gripe: la OMS responde a las críticas. (Nota informativa No 21). Documento consultado el 14/03/12. Disponible en: http://www.who.int/csr/disease/swineflu/notes/briefing 20100610/es/index.html

39. Ordaz, P. (2009a, 26 de Abril). México se pone la mascari1la. El País, pág. 42.

40. Ordaz, P. (2009b, 26 de Abril). Alarma mundial por el virus. El País, pag. 1.

41. Orr, E., Sagi, S. y Bar-On, D. (2000). Social representations in use: Israeli and Palestinian high school students'collective coping and defence. Papers on Social Representations-Online, 9, 2.1-2.20. Documento consultado el 4/11/12. Disponible en: http://www.psych.lse.ac.uk/ psr/PSR2000/9_2Orr.pdf

42. Pan, Z. y Kosicki, G. (2001). Framing as Strategic Action. En S.Reese, O.Gandy, A.Grant, (Comp.), Framing Public Life: Perspectives on Media and our understanding of the social world (pp. 35-66). Mahwah: Lawrence Erlbaum.

43. Pan, Z. y Kosicki, G.M. (2005). Framing and the understanding of citizenship. En S. Dunwoody, L B. Becker, G. M. Kosicki, eta D. M. McLeod (Comp.), The evolution of key communication concepts: Honoring Jack M. McLeod (167207). Cresskill: Hampton Press.

44. Pennebaker, J.W. y Harber, K. (1993). A social stage model of collective coping: The Persian Gulf and other natural disasters. Journal of Social Issues, 49, 125-145. http://dx.doi.org/10.1111/j.1540-4560.1993.tb01184.x

45. Piña, K. (2009, 29 de abril). Muere pequeña dada de alta en clínicas del GDF. El Universal, pág. 6

46. Reese, S. D., Gandy, Jr., O. H. y Grant, A. E. (2001). Framing public life: Perspectives on media and our understanding of the social world. Mahwah, NJ: Lawrence Erlbaum Associates.

47. Reinert, M. (1996). Alceste (Version 3.0). Toulouse: Images.

48. Reinoso, J. (2009, 4 de Mayo). Vigilancia de excepción. El País, pág. 39.

49. Rocamora Villena, V. (2012). De la Comunicación de Riesgos a la Comunicación de Crisis. La OMS en el caso de la gripe A (H1N1). Comunicación presentada al III Congreso Internacional de la AE-IC “Comunicación y Riesgo". Tarragona, 18-20 enero (paper).

50. Rodríguez, R. (2009, 15 de octubre). Más de mil 300 casos de A H1N1 en un día. El Universal, pag. 11

51. Rosas, V. (2009, 2 de mayo). Crece rechazo en el mundo a mexicanos. El Universal, pag. 1

52. Rosenbrock, R., Dubois-Arber, F., Moers, M., Pinell, P., Schaeffer, D. y Setbon, M. (2000). The normalization of AIDS in Western European countries. Social Science and Medicine, 50, 1607-1629. http://dx.doi.org/10.1016/ S0277-9536(99)00469-4

53. Scheufele, D. (2000). Agenda-setting, priming and framing revisited: Another look at cognitive effects of political communication. Mass Communication and Society, 3, 297-316. http://dx.doi.org/10.1207/S15327825MCS0323 07

54. Scheufele, D. y Tewksbury, D. (2007). Framing, Agenda Setting, and Priming: The evolution of Three Media 
Effects Models. Journal of Communication, 57, 9-20. http://dx.doi.org/10.1111/j.0021-9916.2007.00326.x

55. Semetko, H. y Valkenburg, P. (2000). Framing European Politics: A Content Analysis of Press and Television News. Journal of Communication, 50, 93-109. http://dx.doi.org/10.1111/j.1460-2466.2000.tb02843.x

56. Setbon, M. (2000). La normalisation paradoxale du sida [The paradoxical normalization of AIDS]. Revue Française de Sociologie, 41, 61-78. http://dx.doi.org/10.2307/3322654

57. Sperber, D. y Wilson, D. (1986). Relevance-comunication and cognition. Oxford: Blackwell.

58. Tanner, A., Friedman, D.B., Koskan, A. y Barr, D. (2009) Disaster communication on the internet: A focus on Mobilising Information. Journal of Health Communication, 14, 741-755. http://dx.doi.org/10.1080/10810730903295542

59. Valencia, J., Gil De Montes, L., Ortiz, G., Larrañaga, M. y Idoyaga, N. (2010). Enmarcamiento y el rechazo o aceptación de los aspectos generales y específicos de la Ley de igualdad de Género en España: representaciones sociales y regulaciones normativas. Revista de Psicología Social, 25, 145-155. http://dx.doi.org/10.1174/021347410791063769

60. Valkenburg, P.M., Semetko, H. A. y De Vreese, C.H. (1999). The effects of news frames on readers' thoughts and recall. Communications Research, 26, 550-569. http://dx.doi.org/10.1177/009365099026005002
61. Vicente, M. y López, P. (2009). Resultados actuales de la investigación sobre framing: sólido avance internacional y arranque de la especialidad en España. ZER, 14, 13-34.

62. Voelklein, C. y Howard, C. (2005). A Review of Controversies about Social Representations Theory: A British Debate. Culture \& Psychology, 11, 431 - 454. http://dx.doi.org/10.1177/1354067X05058586

63. Wagner, W. (1998). Social representations and beyond-brute facts, symbolic coping and domesticated worlds. Culture and Psychology, 4, 297-329. http://dx.doi.org/10.1177/1354067X9800400302

64. Wagner, W. y Hayes, N. (2005). Everyday discourse and common sense: The theory of social representations. Basingstoke: Palgrave Macmillan.

65. Wagner, W., Kronberger, N. y Seifert, F. (2002). Collective symbolic coping with new technology: Knowledge, images and public discourse. British Journal of Social Psychology, 41, 323-343. http://dx.doi. org/10.1348/014466602760344241

Fecha de recepción: 18 de junio de 2012

Fecha de recepción de la versión modificada: 18 de septiembre de 2012 Fecha de aceptación: 2 de noviembre de 2012 